\title{
Two-thirds of seniors in long-term care take 10 or more drugs: $\mathrm{CIHI}$
}

$\mathrm{S}$ eniors living in long-term care facilities take far more prescription drugs, and more potentially dangerous drugs, than those living at home, the Canadian Institute for Health Information (CIHI) reveals in a new report.

Nearly two-thirds $(60.9 \%)$ of Canadians aged 65 and older who live in long-term care (LTC) facilities take 10 or more different prescription drugs. This is more than double the percentage among seniors who live at home (26.1\%), according to the May 1 report, Drug Use Among Seniors on Public Drug Programs in Canada, 2012.

The report also indicates higher use of psychotropic drugs among LTC residents, as well as drugs deemed "potentially inappropriate" for seniors due to an elevated risk of adverse effects, lack of efficacy or the availability of safer alternatives.

Specifically, seniors in LTC facilities used benzodiazepines, antidepressants and antipsychotics at rates two, three and nine times higher, respectively, than seniors living in the community.

These differences can partly be explained by demographics, says Michael Gaucher, director of pharmaceuticals and health workforce information services at CIHI. "Seniors in long-term care have more chronic conditions, they're generally older and require more care, and quite often these conditions have advanced, so we expected they would require more drugs."

Without diagnostic data, it's impossible to tell how much of this prescribing is appropriate, he notes. However, "we can show trends in the data that can perhaps inform some discussions around how these drugs are being used."
Gaucher cites the higher rates of psychotropic use in LTC facilities as cause for potential concern, given the increased risks associated with these drugs.

Six of the top 10 potentially inappropriate chemicals prescribed to seniors in

among seniors also increases the risk of adverse effects and drug interactions.

The report highlights the complexity of prescribing for seniors, says Dr. Paula Rochon, a geriatrician and vicepresident of research at Women's College Hospital in Toronto, Ontario. "When people have behaviours associated with things like dementia, which is so common in LTC settings, there is no single drug out there without risk."

"Research has demonstrated variation in the rate of antipsychotic prescribing between facilities, while the residents appear similar," she adds. "This suggests the opportunity to further explore the use of nonpharmacological approaches."

Such approaches might include, "creating a calming environment and areas in which people can wander safely, using music and having activities available," says Rochon.

CIHI's report sets a baseline for tracking the number and types of drugs used by LTC residents in comparison to seniors living at home. The findings are based on data sub-

2012 fell into this category, with the antianxiety drug lorazepam topping the list. At fifth and ninth on the list, respectively, antipsychotics such as quetiapine and risperidone are also commonly used to treat the behavioural and psychological symptoms of dementia, including delusion, aggression and agitation.

According to CIHI, use of psychotropic drugs is linked to an increased number of falls among seniors, and in the case of antipsychotics prescribed to elderly patients with dementia, may be associated with an increased risk of death. Use of multiple medications mitted to public drug programs in eight provinces - British Columbia, Alberta, Saskatchewan, Manitoba, Ontario, New Brunswick, Nova Scotia and Prince Edward Island - as well as one federal drug program managed by First Nations and Inuit Health.

Seniors living in PEI used the fewest drugs on average, with just $13.5 \%$ using 10 or more medications. Those covered by the federal government used the most on average, with $53.7 \%$ using 10 drugs or more. — Lauren Vogel, CMAJ

CMAJ 2014. DOI:10.1503/cmaj.109-4797 